\title{
Incidence of Chlamydia trachomatis infections in the provinces of Novara and VCO: one year of observations
}

\author{
Stefano Andreoni, Maria Giuliana Brunelli, Anna Camaggi, Monia Mantovani, Paola Ruzza, \\ Gian Lorenzo Molinari, Vesselina Kroumova, Giacomo Fortina \\ Laboratorio di Microbiologia e Virologia, Azienda Ospedaliero-Universitaria "Maggiore della Carità" di Novara
}

Key words: Chlamydia trachomatis, molecular biology, sexually infections

Incidenza di infezioni da Chlamydia trachomatis nelle province di Novara e VCO: un anno di osservazioni

\section{SUMMARY}

Chlamydia trachomatis infections represent the most common sexually transmitted diseases. The lack of growth of these microorganisms on common media and the roundabout research by immunofluorescence, has meant that, until now, these special-lived intracellular microorganisms have played a role probably underestimated in cases of infections affecting the sexual organs. The introduction of molecular biology has made possible their research in a much more simple, reliable and standardized methods.

The study was carried out on endocervical samples, male urethral swabs and urine samples of men and women, determining the $C$. trachomatis DNA amplified using the BD ProbeTec ET system. This system uses technology SDA (Strand Displacement Amplification - Elongationcrowding of the blanks). The study was conducted from June 2008 to june 2009. Symptomatic and asyntptomatic patients residents in the provinces of Novara and the Verbano-Cusio-Ossola were considered. In this period 784 determinations were made in molecular biology. Chlamydia trachomatis was found in 26 cases equal to a percentage of $3.32 \%$.

While considering these preliminary data, however, we can estimate the rate of detection of $C$. trachomatis as a good starting point, because we have the impression that improved procedures particularly relate to the techniques for sampling and for storage and transport of the sample, can undoubtedly lead to much higher percentages.

Quelle da C. trachomatis (4) rappresentano le più comuni infezioni batteriche a trasmissione sessuale. C. trachomatis è responsabile di importanti infezioni genito-urinarie sessualmente trasmissibili; è una delle maggiori cause di uretrite, cervicite e MIP, nelle donne. Le conseguenze di queste infezioni sono importante causa di gravidanze ectopiche, infertilità e dolore pelvico cronico (2). Negli uomini è causa dal $10 \%$ al $20 \%$ delle uretriti non gonococciche e di più del $50 \%$ dei 150.000 casi di epididimiti (3).

L'impossibilità di crescita di questi microrganismi sui comuni terreni colturali (5) e l'indaginosità delle ricerche in immunofluorescenza, ha fatto sì che, fino ad ora, questi particolari microrganismi a vita intracellulare abbiano rivestito un ruolo probabilmente sottostimato nei casi di infezioni che riguardino la sfera sessuale. D'altra parte, anche le indagini sierologiche si prestano a volte a difficili e non decisive interpretazioni (6). L'introduzione di metodiche in biologia molecolare ha reso possibile la ricerca di $C$. trachomatis in maniera decisamente più semplice, rapida, affidabile e standardizzata (1).

Lo studio è stato effettuato in campioni endocervicali, tamponi uretrali maschili e in campioni di urine di uomini e donne, andando a dosare il DNA amplificato di C. trachomatis utilizzando il kit BD ProbeTec ET C. trachomatis (CT) Amplified DNA Assay. Tale sistema utilizza la tecnologia SDA (Strand Displacement Amplification - allungamentospiazzamento degli spezzoni).

Lo studio è stato condotto nel periodo giugno 2008/giugno 2009 e ha riguardato pazienti sintomatici e no residenti nelle province di Novara e del Verbano-Cusio-Ossola. In tale periodo sono state effettuate 784 determinazioni per C. trachomatis mediante test BD ProbeTec ET C. trachomatis (CT) Amplified DNA Assay, e tali determinazioni hanno riguardato 601 femmine (562 tamponi uretrali/cervicali e 39 urine) e 183 maschi (70 tamponi uretrali, 110 urine e 3 liquidi seminali). La positività totale per $C$. trachomatis è stata di 26 casi pari ad una percentuale del $3.32 \%$.

Per quanto riguarda i maschi, la fascia di età più rappresentativa è stata quella tra i 30-60 anni (127 casi), mentre molto meno rappresentative le altre due fasce di età considerate, 1429 anni (40 casi), > 60 anni $(16$ casi). La positività totale è risultata essere di soli 5 casi $(2.73 \%)$.
Per quanto riguarda le femmine la positività nella fascia di età $15-25$ anni è stata di 7 casi su $90(7.80 \%)$, in quella $26-50$ anni è stata di 10 su $460(2.20 \%)$ mentre in quella oltre 50 anni è stata di 1 su $51(2.20 \%)$.

Pur considerando questi dati preliminari (ci si ripromette infatti di valutare in un prossimo studio eventuali differenze basate sulle diverse modalità di prelievo), possiamo tuttavia valutare la percentuale di rilevazione di C. trachomatis come un buon punto di partenza, perché si ha l'impressione che un miglioramento delle procedure legate in particolare alla tecnica di prelievo, nonché ad una corretta conservazione e trasporto del campione, e soprattutto mirate a determinate fasce d'età, possano indubbiamente portare a percentuali decisamente più significative. A differenza di quanto accade per altre patologie i costi maggiori dell'infezione da C. trachomatis sono legati ai casi non diagnosticati e derivano dalla terapia delle complicanze e dei casi secondari che ne derivano. Appare evidente dunque come il controllo della diffusione delle infezioni da C. trachomatis rappresenti una priorità sanitaria, e come questo controllo debba essere indirizzato in particolar modo verso soggetti femminili con età al di sotto dei 25 anni. A tale scopo diventa indispensabile, proprio perché ci si rivolge per lo più a persone asintomatiche, disporre di test non invasivi, e quindi facilmente accettabili, ed allo stesso tempo altamente sensibili e specifici.

\section{BIBLIOGRAFIA}

1. Bas S, Muzzin P, Ninet B, Bornand JE, Scieux C, Vischer TL. Chlamydial serology: comparative diagnostic value of immunoblotting, microimmunofluorescence test, and immunoassays using different recombinant proteins as antigens. J Clin Microbiol 2001 Apr; 39(4): 1368-77.

2. Bebear C, de Barbeyrac B. Genital Chlamydia trachomatis infection. Clin Microbiol Infect 2009; 15(1): 4-10.

3. Bjarthing C, Osser S, Johnsson A, Persson K. Clinical manifestations and epidemiology of the new genetic variant of Chlamydia trachomatis. Sex Transm Dis 2009; 36(9): 529-35.

4. Cevenini R, Donati M, Sembri V. Chlamydia trachomatis-the agent. Best Pract Res Clin Obstet Gynaecol 2002; 16(6): 761-73.

5. Churokov AA, Kulichenko AN, Kzakova ES, Serebrianik NE, Suvorov AP, Kutyrev W, Glybochko PV. Laboratory diagnostics of urogenital clamidiosis. Klin Lab Diagn 2005 Feb; (2): 43-7 Russian.

6. Tuuminen T, Palomaki P, Paavonen J. The use of serologic test for the diagnosis of chlamydial infections. J Microbiol Methods 2000 Nov; 42 (3): 265-79.

\section{Corresponding author: Stefano Andreoni}

Laboratorio di Microbiologia e Virologia - Azienda Ospedaliero-Universitaria "Maggiore della Carità"

Corso Mazzini 18 - 28100 - Novara - Tel.: 0321 3733091; Fax: 03213733588

E-mail: stefano.andreoni@maggioreosp.novara.it 